Quim. Nova, Vol. 28, No. 1, 46-49, 2005

\title{
SÍNTESE E CARACTERIZAÇÃo DE $\alpha$-FOSFATO DE ZIRCÔNIO(IV) CONTENDO AGREGADOS DE COBRE METÁLICO
}

\author{
Alexilda Oliveira de Souza* \\ Departamento de Estudos Básicos e Instrumentais, Universidade Estadual do Sudoeste da Bahia, Praça Primavera, 40, \\ Primavera, 45700-000 Itapetinga - BA \\ Maria do Carmo Rangel \\ Departamento de Físico-Química, Instituto de Química, Universidade Federal da Bahia, Campus Universitário de Ondina, \\ 40170-290 Salvador - BA \\ Oswaldo Luiz Alves \\ Departamento de Química Inorgânica, Instituto de Química, Universidade Estadual de Campinas, CP 6154, $13084-971$ \\ Campinas - SP
}

Recebido em 27/1/04; aceito em 7/7/04; publicado na web em 12/11/04

\begin{abstract}
SYNTHESIS AND CHARACTERIZATION OF $\alpha$-ZIRCONIUM (IV) HYDROGENPHOSPHATE CONTAINING METALLIC COPPER CLUSTERS. The $\alpha$-zirconium (IV) hydrogenphosphate $(\alpha-\mathrm{ZrP})$ has received great attention in the last years due to its properties like ion exchange, intercalation, ionic conductivity and catalytic activity. This work reports a method to produce metallic copper clusters on $\alpha-\mathrm{ZrP}$ to be used as catalysts in petrochemical processes. It was found that the solids were non-crystalline regardless of the uptake of copper and the reduction. The specific surface area increased as a consequence of the increase of the interlayer distance to accept the copper ions between the layers. During the reduction, big clusters of copper $(0,5-11 \mu)$ with different sizes and shapes were produced.
\end{abstract}

Keywords: $\alpha$-zirconium(IV) hydrogenphosphate; copper(II) exchanged zirconium phosphat; layered zirconium phosphates.

\section{INTRODUÇÃO}

$\mathrm{O} \alpha$-fosfato de zircônio(IV), $\alpha$-ZrP, é um dos membros mais estudados da família dos sólidos ácidos lamelares. Esses compostos vêm despertando grande interesse científico nos últimos anos por apresentarem diversas propriedades úteis, tais como troca iônica, intercalação, atividade catalítica e condutividade iônica ${ }^{1,2}$.

Os fosfatos de zircônio(IV) podem ser obtidos como géis ou sólidos em diferentes estágios de cristalinidade, como sólidos altamente cristalinos, com propriedades de troca iônica variadas em função da cristalinidade. Os géis podem ser obtidos pela adição de um sal solúvel de $\mathrm{Zr}(\mathrm{IV})$ ao ácido fosfórico, enquanto os compostos cristalinos podem ser preparados através do refluxo desses géis em ácido fosfórico. Variando-se a concentração do ácido e o tempo de refluxo, pode-se preparar materiais com diferentes graus de cristalinidade. Os compostos cristalinos podem ser obtidos como sólidos lamelares de dois tipos: o $\mathrm{Zr}\left(\mathrm{HPO}_{4}\right)_{2} \cdot \mathrm{H}_{2} \mathrm{O}$ com espaço interlamelar de $7,6 \AA$ e o $\mathrm{Zr}\left(\mathrm{HPO}_{4}\right)_{2} \cdot 2 \mathrm{H}_{2} \mathrm{O}$ com espaço interlamelar de $12 \AA$, denominados $\alpha-\mathrm{ZrP}$ e $\gamma-\mathrm{ZrP}$, respectivamente ${ }^{3-5}$.

Entre esses materiais, o $\alpha$-ZrP é o mais estudado sendo sua estrutura cristalina primeiramente proposta por Clearfield e $\mathrm{Smith}^{6}$, através de estudos de difração de raios X. Em trabalho posterior, Albertson e colaboradores determinaram a posição dos átomos de hidrogênio através de difração de nêutrons ${ }^{7}$. A composição química do $\alpha-Z r P$ cristalino é caracterizada por uma razão $\mathrm{P} / \mathrm{Zr}=2$, que leva a um composto estequiométrico formulado como $\mathrm{Zr}\left(\mathrm{HPO}_{4}\right)_{2} \cdot \mathrm{H}_{2} \mathrm{O}$, apresentando estrutura lamelar. Cada lamela do $\alpha-Z r P$ é formada por átomos de zircônio num plano e ligados aos grupos fosfatos. Três átomos de oxigênio de cada fosfato tetraédrico estão ligados a três átomos de zircônio, de modo que cada um destes está octaedricamente coordenado a seis oxi-

*e-mail: alexilda@uesb.br gênios de seis grupos fosfatos distintos. O quarto átomo de oxigênio de cada grupo fosfato liga-se a um próton, que pode ser trocado por uma variedade de cátions, principalmente metais alcalinos, alcalinoterrosos e metais de transição divalentes. Após a troca iônica, geralmente ocorre uma expansão do espaço interlamelar para acomodar os íons e as moléculas de água ${ }^{8}$.

Neste trabalho, foi desenvolvido um método de preparação para produzir um compósito de cobre metálico/fosfato de zircônio, visando a obtenção de novos catalisadores para a indústria química e petroquímica. As espécies $\mathrm{Cu}^{2+}$ foram introduzidas na estrutura do $\alpha$-ZrP, através de troca iônica, e os sólidos foram posteriormente reduzidos para formar os agregados de cobre metálico.

\section{PARTE EXPERIMENTAL}

$\mathrm{O} \alpha$-fosfato de zircônio(IV) foi obtido via processo sol-gel, reagindo-se uma solução $0,5 \mathrm{~mol} / \mathrm{L}$ de oxicloreto de zircônio com ácido fosfórico concentrado. O gel formado permaneceu no meio reacional por $24 \mathrm{~h}$, depois foi isolado por centrifugação e lavado com água deionizada até a eliminação dos íons cloretos e pH em torno de 4 . O material obtido foi refluxado por $48 \mathrm{~h}$, em uma solução 1,0 mol/L de ácido fosfórico. Após o refluxo, o gel foi isolado por centrifugação e lavado com água deionizada até se obter pH em torno de 4. Em seguida, o material foi seco a $35^{\circ} \mathrm{C}$ até a obtenção de massa constante $\mathrm{e}^{9,10}$.

Com o produto da etapa anterior foi realizada a troca iônica, submetendo-se o material ao refluxo por $24 \mathrm{~h}$ numa solução aquosa de acetato de cobre(II) 0,1 mol/L. Em seguida, o material (cerca de $2 \mathrm{~g}$ ) foi acondicionado em uma cela de quartzo e aquecido, a uma taxa de $10{ }^{\circ} \mathrm{C} \mathrm{min}^{-1}$, até $500{ }^{\circ} \mathrm{C}$, permanecendo nesta temperatura por 1 h. Empregou-se uma mistura contendo $10 \% \mathrm{CO}, 10 \% \mathrm{CO}_{2}, 20 \% \mathrm{~N}_{2}$ e $60 \% \mathrm{H}_{2}\left(40 \mathrm{~mL} \mathrm{~min}^{-1}\right)$. 
O teor de zircônio nos sólidos foi determinado por gravimetria, usando-se o método do Cupferon ${ }^{11} \mathrm{em}$ amostras previamente dissolvidas em uma solução $20 \%$ de ácido sulfúrico. Na determinação do conteúdo de fósforo, as amostras foram oxidadas com ácido nítrico e, em seguida, analisadas na forma de fosfomolibdato ${ }^{12}$, empregando-se um espectrofotômetro Hitachi modelo U-2000 Hitachi. A quantidade de cobre nos sólidos foi analisada por absorção atômica, usando-se um equipamento Perkin Elmer modelo 5000 e amostras previamente dissolvidas em ácido fluorídrico concentrado.

As medidas de difratometria de raios $\mathrm{X}$ das amostras em pó foram realizadas em um difratômetro Shimadzu modelo XD3A, composto de um goniômetro modelo VG-108 R e um tubo gerador de raios-X modelo A-40, utilizando a radiação $\operatorname{CuK} \alpha(\lambda=1,5418 \AA$ ) e filtro de níquel. Foram usadas as seguintes condições na obtenção dos difratogramas: voltagem de $35 \mathrm{kV}$, corrente de $25 \mathrm{~mA}$ e varredura de $2 \%$ min. A preparação das amostras consistiu na aplicação de uma camada compacta do pó sobre um porta-amostra de alumínio.

Os espectros no infravermelho foram obtidos em um espectrômetro com transformadas de Fourier Perkin Elmer 1600 FTIR, na região de 4000-400 $\mathrm{cm}^{-1}$, resolução de $4 \mathrm{~cm}^{-1}$ e métodos de amostragem em suspensão de Nujol, entre janelas de $\mathrm{KBr}$.

Na obtenção dos espectros Raman, utilizou-se o equipamento Renishaw Raman Imaging Microscope System 3000, acoplado a um microscópio óptico com resolução de $1,5 \mu \mathrm{m}$ e laser de $\mathrm{He}-\mathrm{Ne}(\lambda=$ $633 \mathrm{~nm}$ ). A amostragem foi feita depositando-se o pó sobre uma lâmina de vidro.

As áreas superficiais específicas foram medidas utilizando-se um equipamento Micromeritcs TPD/TPR 2900 Analyser e uma massa de sólido de cerca de $0,5 \mathrm{~g}$. As amostras foram acondicionadas numa cela de vidro e aquecidas em atmosfera de nitrogênio a $10^{\circ} \mathrm{C} / \mathrm{min}$ até $160{ }^{\circ} \mathrm{C}$, durante $1 \mathrm{~h}$. Após o período de ativação, executou-se a análise utilizando uma mistura $30 \% \mathrm{~N}_{2} / \mathrm{He}$ e o método BET.

As micrografias eletrônicas de varredura foram obtidas em um microscópio JEOL JSM T-300. Na preparação das amostras, o pó foi depositado sobre fita adesiva de dupla face, fixada em um portaamostra de carbono, na forma de uma camada fina, que foi coberta com carbono, utilizando um metalizador Bal-Tec MED 020.

\section{RESULTADOS E DISCUSSÃO}

A partir dos difratogramas apresentados na Figura 1, foi possível observar padrões de difração de raios $\mathrm{X}$ típicos de fosfatos de zircônio não-cristalinos. $\mathrm{O}$ difratograma da amostra aquecida em atmosfera redutora apresentou dois picos em $2 \theta=43$ e $49^{\circ}$, atribuídos ao cobre metálico, por comparação com o difratograma de um padrão de cobre metálico.

A Figura 2 mostra os espectros de FTIR das amostras obtidas. A banda na faixa de $1075-980 \mathrm{~cm}^{-1}$, que aparece em todos os casos, pode ser atribuída às vibrações do grupo fosfato ${ }^{13}$. Essas bandas são largas e mal definidas confirmando a estrutura não-cristalina desses materiais, observada por difração de raios X. No espectro do fosfato de zircônio puro (amostra Z), nota-se a presença de uma banda a $2460 \mathrm{~cm}^{-1}$, devido à vibração de estiramento da ligação $\mathrm{P}-\mathrm{OH}^{13}$. Essa banda apareceu menos intensa no espectro dos materiais tratados com cobre, indicando a formação de entidades $\left[\mathrm{mP}^{-} \mathrm{O}^{-}\right]_{2} \mathrm{Cu}^{2+}$. A amostra aquecida em atmosfera redutora não apresentou as bandas situadas na região de $3400 \mathrm{~cm}^{-1}$ e em $1640 \mathrm{~cm}^{-1}$ associadas aos modos de estiramento e deformação da ligação $\mathrm{OH}$, característicos da molécula de água, indicando que os compostos foram completamente desidratados durante o tratamento térmico.

O espectro Raman, na região de 200 a $2000 \mathrm{~cm}^{-1}$, da amostra aquecida em atmosfera redutora, não pôde ser obtido em função da elevada fluorescência que esse material apresentou nessa região. Nos

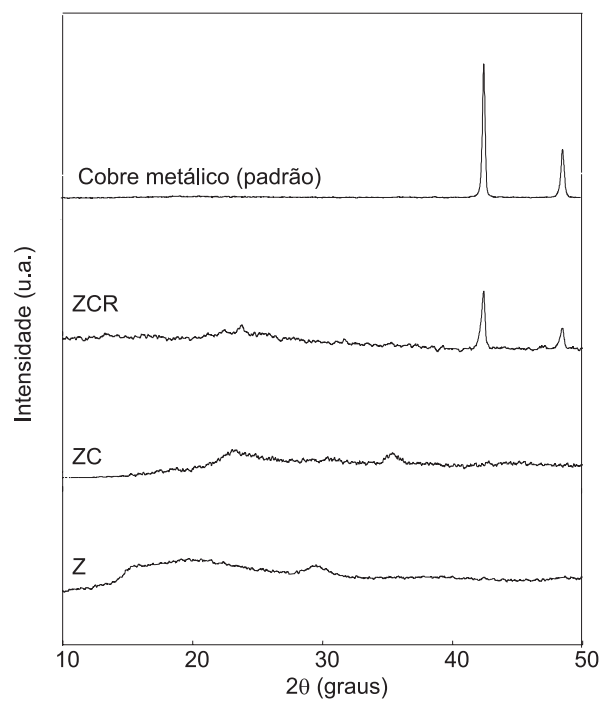

Figura 1. Difratogramas de raios $X$ do fosfato de zircônio (Z), fosfato de zircônio trocado com cobre (ZC), fosfato de zircônio trocado com cobre e aquecido a $500{ }^{\circ} \mathrm{C}$ em atmosfera redutora $(\mathrm{ZCR})$ e padrão de cobre metálico

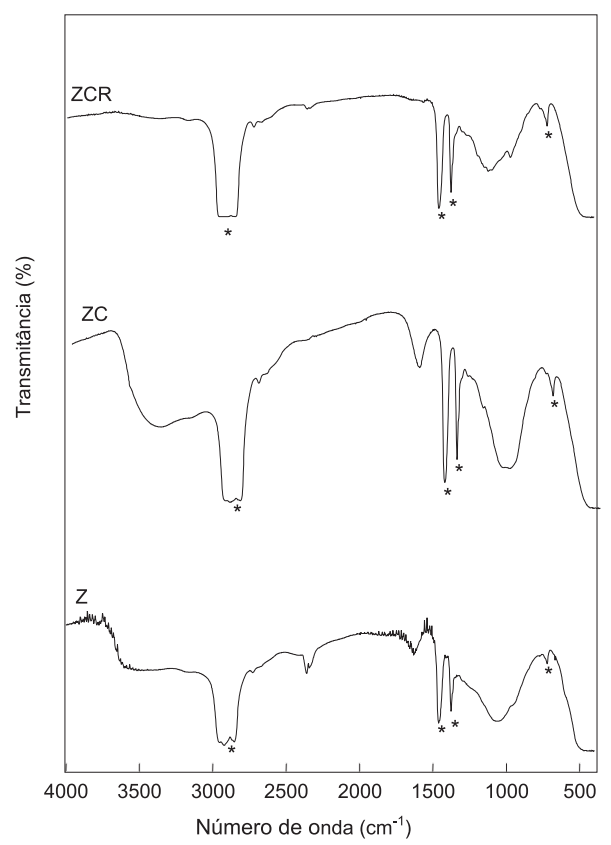

Figura 2. Espectros no infravermelho do fosfato de zircônio (Z), fosfato de zircônio trocado com cobre $(Z C)$ e fosfato de zircônio trocado com cobre e aquecido a $500{ }^{\circ} \mathrm{C}$ em atmosfera redutora (ZCR). As bandas assinaladas com * referem-se ao dispersante Nujol

espectros do fosfato de zircônio puro e naquele trocado com cobre, observou-se uma banda na região de estiramento P-O (1150-1000 $\left.\mathrm{cm}^{-1}\right)^{13}$. O espectro do material trocado com cobre mostrou um alargamento dessa banda, em relação ao fosfato original, sugerindo um aumento da desordem na estrutura, devido à entrada do íon cobre hidratado. Os espectros Raman obtidos estão ilustrados na Figura 3.

Os resultados de análise química (Tabela 1) mostraram que o fosfato de zircônio puro apresentou uma razão $\mathrm{P} / \mathrm{Zr}$ de 1,58 , que é inferior ao valor estequiométrico de 2 e correspondente à fórmula $\mathrm{Zr}(\mathrm{OH})_{0,84}\left(\mathrm{HPO}_{4}\right)_{1,58}$. A troca iônica com cobre praticamente não alterou a razão $\mathrm{P} / \mathrm{Zr}$ e o fosfato trocado apresentou uma razão $\mathrm{P} / \mathrm{Zr}$ de 1,56 , indicando que o fosfato de zircônio permaneceu estável em meio de acetato de cobre sob refluxo. O processo de troca levou à 


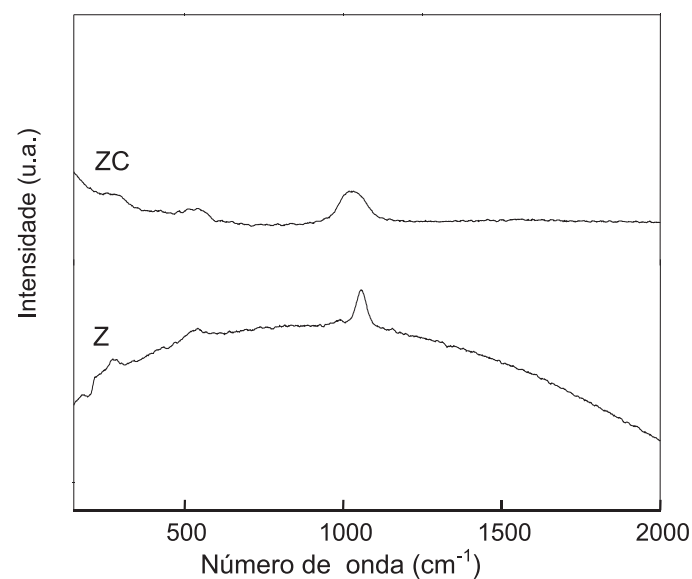

Figura 3. Espectros Raman do fosfato de zircônio (Z) e fosfato de zircônio trocado com cobre $(Z C)$

incorporação de 6,49 meq.g ${ }^{-1}$ do metal, que é próximo ao valor correspondente à capacidade de troca iônica do fosfato de zircônio $(6,64$ meq. $\left.\mathrm{g}^{-1}\right)^{1}$. Isto pode ser atribuído à baixa cristalinidade dos sólidos. Como observado em trabalhos anteriores, a troca com cobre não ocorre facilmente na estrutura do $\alpha-\mathrm{ZrP}$ porque o íon cobre não pode passar pelas estreitas cavidades interplanares; entretanto, a incorporação desse metal torna-se significativa, à medida que o material se torna menos cristalino e essas distâncias se tornam maiores ${ }^{14-16}$.

Tabela 1. Resultados de análise química do fosfato de zircônio (Z) e fosfato de zircônio trocado com cobre (ZC)

\begin{tabular}{lccccc}
\hline Amostra & $\begin{array}{c}\% \mathrm{P} \\
\pm 0,02\end{array}$ & $\begin{array}{c}\% \mathrm{Zr} \\
\pm 0,04\end{array}$ & $\begin{array}{c}\mathrm{P} / \mathrm{Zr} \\
\% \mathrm{Cu} \\
\pm 0,01\end{array}$ & $\begin{array}{c}\text { meq.g } \\
\pm 0,01\end{array}$ \\
\hline $\mathrm{Z}$ & 16,70 & 31,12 & 1,58 & - & - \\
$\mathrm{ZC}$ & 16,48 & 30,94 & 1,56 & 18,21 & 6,49 \\
\hline
\end{tabular}

De acordo com os resultados de área superficial específica (Tabela 2), observou-se que os sólidos obtidos apresentaram áreas superficiais específicas baixas, esses valores são típicos de fosfatos de zircônio, que tendem a formar partículas grandes e não porosas ${ }^{8}$. Nota-se que a área superficial específica aumentou devido à incorporação do cobre, provavelmente devido à separação entre as lamelas para acomodar as espécies $\mathrm{Cu}^{2+10}$.

A Figura 4 ilustra as micrografias eletrônicas de varredura do fosfato de zircônio puro, trocado com cobre e tratado em atmosfera redutora. $\mathrm{O}$ fosfato de zircônio apresenta uma textura rugosa, enquanto que o material trocado com cobre mostra uma superfície lisa, sugerindo que os agregados de cobre se formam ao longo das fissuras e outras regiões onde existem espaços dentro do cristal. Após o aquecimento em atmosfera redutora, são observados agregados grandes (0,5-11 $\mu \mathrm{m})$ de cobre metálico na superfície, com tamanhos e formas variadas, com tendência à morfologia esférica.

Tabela 2. Áreas superficiais específicas $(\mathrm{Sg})$ do fosfato de zircônio (Z), fosfato de zircônio trocado com cobre (ZC) e fosfato de zircônio trocado com cobre e tratado a $500{ }^{\circ} \mathrm{C}$ em atmosfera redutora $(\mathrm{ZCR})$

\begin{tabular}{cc}
\hline Amostra & $\mathrm{Sg}\left(\mathrm{m}^{2} \mathrm{~g}^{-1}\right)$ \\
\hline $\mathrm{Z}$ & 2,0 \\
$\mathrm{ZC}$ & 4,3 \\
$\mathrm{ZCR}$ & 4,6 \\
\hline
\end{tabular}

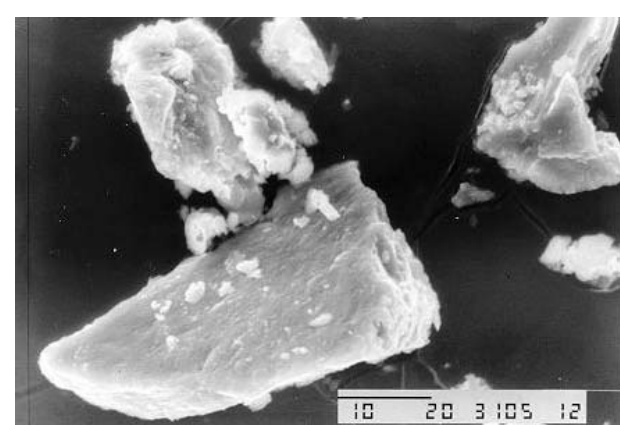

(a) Z

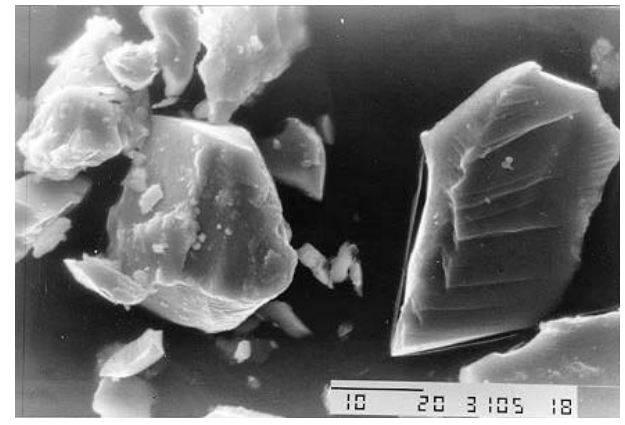

(b) ZC

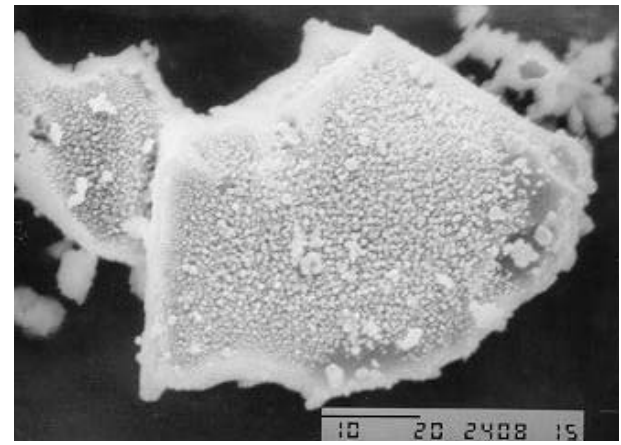

(c) ZCR

Figura 4. Micrografias eletrônicas de varredura do (a) fosfato de zircônio puro $(\mathrm{Z}),(b)$ fosfato trocado com cobre $(\mathrm{ZC})$ e (c) fosfato de zircônio trocado com cobre e aquecido em atmosfera redutora (ZCR)

\section{CONCLUSÕES}

$\alpha$-fosfato de zircônio(IV) não cristalino, correspondente à fórmula $\mathrm{Zr}(\mathrm{OH})_{0.84}\left(\mathrm{HPO}_{4}\right)_{1,58}$, e obtido por refluxo de um gel de zircônio com ácido fosfórico, pode ser modificado por troca iônica com solução de acetato de cobre e posterior tratamento térmico em atmosfera redutora. No material trocado com cobre e submetido à redução, há a formação de um compósito fosfato de zircônio/cobre metálico, no qual o cobre metálico está distribuído na superfície do fosfato na forma de agregados com tamanhos e formas variadas.

\section{AGRADECIMENTOS}

Os autores agradecem ao CNPq, FAPESP e FINEP pelo apoio financeiro.

\section{REFERÊNCIAS}

1. Clearfield, A.; Inorganic Ion Exchange Materials, Ed. CRC Press: Boca Raton, Florida, 1982. 
2. Alamo, J.; Roy, R. J.; Mater. Sci. 1986, 21, 444.

3. Alamo, J.; Solid State Ionics. 1993, 63, 547.

4. Komarneni, S.; Int. J. High Technol. Ceram. 1998, 4, 31.

5. Zsinka, L.; Szirte, L.; So, L. V.; J. Thermal. Anal. Calorim. 1978, 14, 245.

6. Clearfield, A.; Smith, G. D.; Inorg. Chem. 1969, 8, 431.

7. Albertson, A.; Oskarsson, A.; Tellgren, R.; Thomas, J. O.; J. Phys, Chem. 1977, 81, 1534

8. Alberti, G.; Casciola, M.; Constantino, U.; Vivani, R.; Adv. Mater. 1996, 8, 291.

9. Clearfield, A.; Kalnins, J. M.; J. Inorg. Nucl. Chem. 1976, 38, 849.

10. Clearfield, A.; Stynes, J. A.; J. Inorg. Nucl. Chem. 1964, 26,117.
11. Elving, P. J.; Olson E. C.; Anal. Chem. 1955, 27, 1817.

12. Bernhardt, D. N.; Wreath, A. R.; Anal. Chem. 1955, 27, 440.

13. Horsley, S. E.; Nowell, D. V.; Stewart, D. T.; Spectrochm. Acta 1974, 30, 535.

14. Clearfield, A.; Duax, W. L.; Medina, A. S.; Smith, G. D.; Thomas, J. R. J.; Phys. Chem. 1969, 73, 3424.

15. Ahrland, S.; Albertsson, J.; Oskarsson, A.; Niklasson, A.; J. Inorg. Nucl. Chem. 1970, 32, 2069.

16. Ahrland, S.; Bjork, N., Blessing, R. H.; Herman, R. G.; J. Inorg. Nucl. Chem. 1974, 36, 2377. 